\title{
Study of Efficacy of the Use of Peri-Operative Pregabalin and Dexamethasone on Post-Operative Pain in Patients undergoing Lumbar Laminectomy
}

\author{
Mohammed Ahmed El-Feky, Wael Mohammed El-Mahdi and \\ Bahaa Mohamed Abd El-Raouf
}

\author{
Department of Anesthesia and Intensive Care, Faculty of Medicine, Al-Azhar University \\ Corresponding author: Bahaa Mohamed Abd El-Raouf, dr.bahaa007@gmail.com
}

\begin{abstract}
Objective: This study aimed at studying the efficacy of the peri-operative use of pregabalin and dexamethasone for post-operative pain relief in patients undergoing lumbar laminectomy.

Methods: It is a prospective randomized controlled study. The patients on whom the study had been carried out fulfilled the following criteria: Age: above 20 years till 60 years, Gender: both females and males, ASA I and II, Patients scheduled to undergo elective lumbar laminectomy without fusion, patients had been randomly categorized into 2 equal groups by closed envelope technique, 30 in each group. Group P: Pregabalin + Placebo: Patients had been orally given single dose of $150 \mathrm{mg}$ of pregabalin dissolved in $10 \mathrm{ml}$ distilled water one hour before the initiation of anesthesia and until the second post-operative day, the doses had been given every 12 hours for a total of 4 doses. Group PD: Pregabalin + Dexamethasone: Patients had been orally given single dose of $150 \mathrm{mg}$ of pregabalin dissolved in $10 \mathrm{ml}$ distilled water one hour before the initiation of anesthesia and until the second post-operative day, the doses had been given every 12 hours for a total of 4 doses. Patients received $16 \mathrm{mg}$ dexamethasone intravenously with induction of anesthesia.

Results: There was a significant difference about the heart rate intra operative and the mean arterial blood pressure intraoperative and postoperatively at different intervals. As regarding the ETCO2 group P had a higher average at baseline. Group PD had a significant lower consumption of pethidine in comparison with group P. However, there was no significant difference observed in the consumption of ketorolac between the two groups. In comparison between the two studied groups, the overall mean satisfaction score was significantly higher among group PD than among group P.
\end{abstract}

Keywords: Dexamethasone, Pregabaline, Pain Management, Lumbar Laminectomy, Anesthesia

\section{INTRODUCTION}

One of the primary reasons for development of health care is the relief of pain. It is well documented that inadequately relieved pain can lead to complications in the postoperative period. Therefore, the pain of surgery must be relieved totally ${ }^{(1)}$.

No single therapy can achieve this goal. Multimodal strategy refers to the simultaneous use of multiple analgesic methods or drugs. A multimodal approach typically deploys interventions such as local anesthesia; a non-steroidal antiinflammatory (NSAID) drug, or an opioid to achieve a combination of analgesic chemotherapy ${ }^{(2)}$.

Pain is defined as "an unpleasant sensory and emotional experience associated with actual or potential tissue damage. The concept of preemptive analgesia (or treating postoperative pain by preventing the establishment of central sensitization) seems very logical ${ }^{(3)}$.

It has been suggested that performing neural blockade with local anesthetics before the surgical incision may prevent the nociceptive input from altering the excitability of the central nervous system ${ }^{(4)}$.

\section{AIM OF THE WORK}

This study aimed at studying the efficacy of the peri-operative use of pregabalin and dexamethasone for post-operative pain relief in patients undergoing lumbar laminectomy.

\section{PATIENTS AND METHODS}

After approval of the local ethical committee and having an informed consent from every patient, this study was carried out at Al-Azhar hospital on 60 patients. The study was approved by the Ethics Board of Al-Azhar University. The patients on whom the study had been carried out fulfilled the following criteria: Age: above 20 years till 60 years. Gender: both females and males. ASA I and II. Patients scheduled to undergo elective lumbar laminectomy without fusion.

Exclusion criteria: Body mass index is more than $35 \mathrm{~kg} / \mathrm{m}^{2}$. Impaired renal or hepatic function. Pre-operative use of antidepressant or anticonvulsants. Patients who have allergy or contraindication to pregabalin or dexamethasone. Patients who received steroids within 24 hours before surgery or during 72 hours after surgery. Patients had been randomly categorized into 2 equal groups by closed envelope technique, 30 in each group, as recommended by the Biostatistics Department in Public Health, Al-Azhar University.

Group P: Pregabalin + Placebo: Patients had been orally given single dose of $150 \mathrm{mg}$ of 
pregabalin dissolved in $10 \mathrm{ml}$ distilled water one hour before the initiation of anesthesia and until the second post-operative day, the doses had been given every 12 hours for a total of 4 doses $(600 \mathrm{mg}$ in $40 \mathrm{ml}$ water). Patients also received $10 \mathrm{ml}$ normal saline intravenously with induction of anesthesia.

Group PD: Pregabalin + Dexamethasone: Patients had been orally given single dose of $150 \mathrm{mg}$ of pregabalin dissolved in $10 \mathrm{ml}$ distilled water one hour before the initiation of anesthesia and until the second post-operative day, the doses had been given every 12 hours for a total of 4 doses (600 $\mathrm{mg}$ in $40 \mathrm{ml}$ water). Patients received $16 \mathrm{mg}$ dexamethasone intravenously with induction of anesthesia $(4 \mathrm{ml}$ dexamethasone added to $6 \mathrm{ml}$ normal saline with total volume $10 \mathrm{ml}$ ).

Measurements: Demographic characteristics, such as age, BMI, sex, physiological score (ASA), were recorded for all patients.

Pre-operative evaluation and preparation: Proper history taking and clinical examination. Routine laboratory investigations include: Complete blood count. Hemostatic profile study: Bleeding time. Clotting time. Prothrombin time. Partial thromboplastin time (PTT). Prothrombin activity. International normalization ratio (INR). Fasting serum glucose level. Serum urea and creatinine levels. Serum SGOT and SGPT levels. Patients had been randomly categorized into 2 equal groups by closed envelope technique 30 in each group:

Group P: Pregabalin + Placebo: Patients had been given single dose of $150 \mathrm{mg}$ of pregabalin dissolved in $10 \mathrm{ml}$ distilled water orally one hour before the initiation of anesthesia and until the second post-operative day, the doses had been given every 12 hours for a total of 4 doses $(600 \mathrm{mg}$ in $40 \mathrm{ml}$ water). Patients had also received $10 \mathrm{ml}$ normal saline intravenously with induction of anesthesia.

Group PD: Pregabalin + Dexamethasone: Patients had also received $16 \mathrm{mg}$ dexamethasone intravenously with induction of anesthesia $(4 \mathrm{ml}$ dexamethasone added to $6 \mathrm{ml}$ normal saline with total volume $10 \mathrm{ml}$ ).

Before induction of general anesthesia, patients had been connected to standard monitoring: Continuous ECG monitoring. Pulse oximeter. NonInvasive arterial blood pressure monitoring: Mean arterial blood pressure (MAP), systolic blood pressure (SAP) and diastolic pressure (DAP). Capnogram for monitoring ETCO2. Post Anesthesia Care Unit: After the end of surgery, the patients had been transferred to the post anesthesia care unit (PACU) for 2 hours.
Postoperative pain management in the ward had been started as soon as the patient experience pain, it included pethidine $50 \mathrm{mg}$ IV and ketorolac $30 \mathrm{mg}$ IV.

\section{RESULTS}

Regarding age, gender, BMI and ASA there was non-significant differences between the two studied groups within each age group. (figure1, 2, 3, 4).
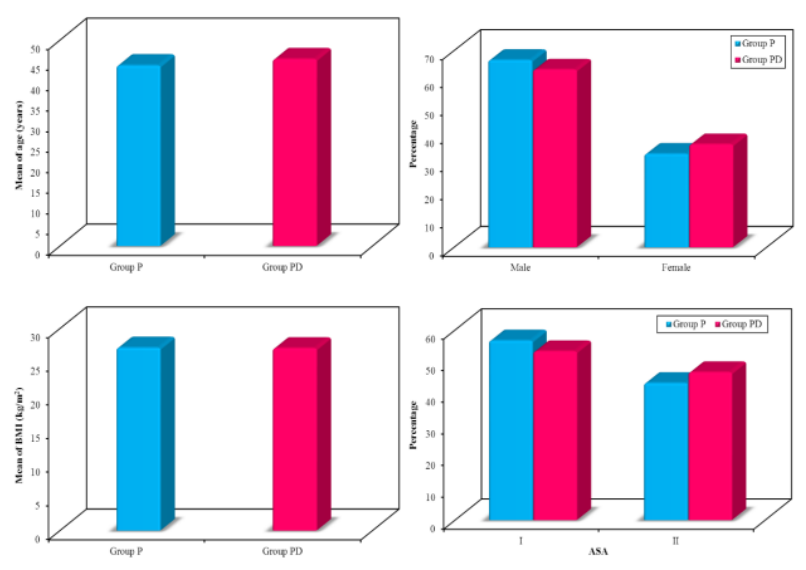

Figures (1): Comparison between the two studied groups regarding age, gender, BMI and ASA.

\section{Comparison between the two studied groups according to heart rate(beats/min) during different periods.}

There was a significant difference as regards the heart rate intra operative at $15 \mathrm{~min}$ ( $\mathrm{p}$ value $<0.001$ ), at $30 \mathrm{~min}$ ( $\mathrm{p}$ value $<0.001$ ), at $45 \mathrm{~min}$ ( $\mathrm{p}$ value $<0.001$ ), at $60 \mathrm{~min}$ ( $\mathrm{p}$ value $<0.001$ ), at $75 \mathrm{~min}(\mathrm{p}$ value $<0.001$ ), and at $90 \mathrm{~min}$ ( $\mathrm{p}$ value $<0.001$ ), at $105 \mathrm{~min}$ ( $\mathrm{p}$ value $<0.001$ ) and at $120 \mathrm{~min}(\mathrm{P}$ value $<0.001)$. In the postoperative period there was significance difference as regard the heart rate at $15 \mathrm{~min}$ ( $\mathrm{p}$ value $<0.001$ ), at 30 $\min$ ( $\mathrm{p}$ value $<0.001$ ), at $45 \mathrm{~min}$ ( $\mathrm{p}$ value $<0.001$ ), at 60 $\min$ ( $\mathrm{p}$ value $<0.001$ ), at $75 \mathrm{~min}$ ( $\mathrm{p}$ value $<0.001$ ), at 90 $\min (\mathrm{p}$ value $<0.001$ ), but there was no significant difference as regards the heart rate at $105 \mathrm{~min}$ ( $\mathrm{p}$ value $=0.077)$ and at $120 \mathrm{~min}(\mathrm{P}$ value $=0.736)$.

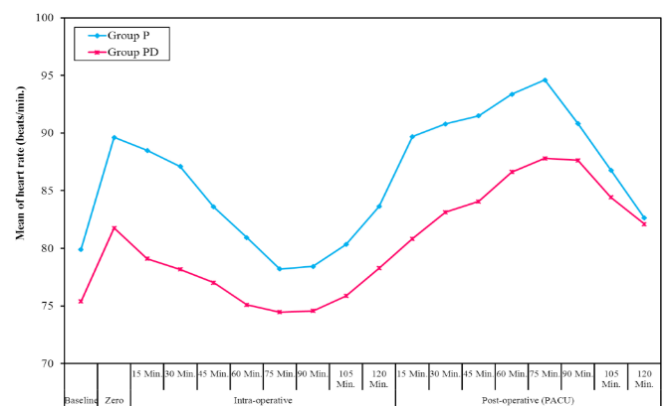

Figure (2): Comparison between the two studied groups according to heart rate. 
Comparison between the two studied groups according to mean arterial blood pressure (mmHg) in different periods:

There was a significant difference as regard the mean arterial blood pressure intraoperative at $15 \mathrm{~min}$ $(\mathrm{p}<0.001)$ at $30 \mathrm{~min}((\mathrm{p}<0.001)$, at $45 \mathrm{~min}(\mathrm{p}$ value $=0.001$ ), at $60 \mathrm{~min}$ ( $\mathrm{p}$ value $=0.023$ ), and at $75 \mathrm{~min}$ ( $\mathrm{p}$ value $=0.037$ ), but there was non significant difference as regard the mean arterial blood pressure intraoperative at $90 \mathrm{~min}$ $(\mathrm{p}=0.18)$. Moreover, there was a significant difference as regard the mean arterial blood pressure intraoperative at $105 \mathrm{~min}$ ( $\mathrm{p}$ value $=0.022$ ) and at $120 \mathrm{~min}$ ( $\mathrm{p}$ value $=0.003$ ). Also in the postoperative period there was significance difference as regard the mean arterial blood pressure at 15 $\min (\mathrm{p}<0.001)$, but there was insignificant difference as regard the mean arterial blood pressure postoperative at 30 $\min$ ( $\mathrm{p}$ value $=0.088$ ), but there was significant difference as regard the mean arterial blood pressure intraoperative at $45 \mathrm{~min}(\mathrm{p}<0.001)$, at $60 \mathrm{~min}(\mathrm{p}<0.001)$, at $75 \mathrm{~min}$ $(p<0.001)$, but there was insignificant difference as regard the mean arterial blood pressure postoperative at $90 \mathrm{~min}(\mathrm{p}$ value $=0.416$ ) at $105 \mathrm{~min}$ ( $\mathrm{p}$ value $=0.701$ ) and at $120 \mathrm{~min}$ ( $\mathrm{p}$ value $=0.567$ )

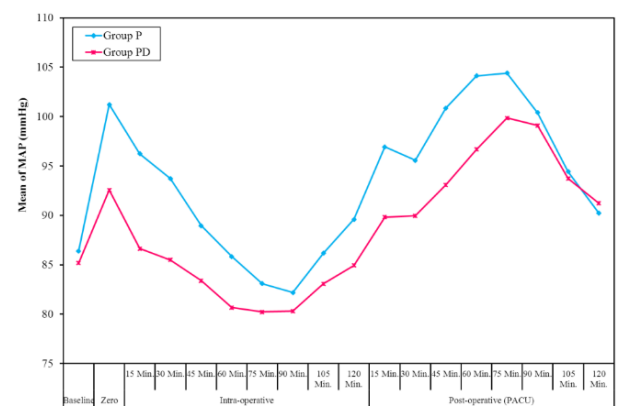

Figure (3): Comparison between the two studied groups according to mean arterial blood pressure/mmHg.

\section{Changes in the oxygen saturation.}

In group $\mathrm{P}$, the mean of arterial oxygen saturation was $97.37 \pm 1 \%$ on admission to operating room, later on there was no significant change in arterial oxygen saturation intraoperatively at 15 minute, at 30 minute, at 45 minute, at 60 minute, at 75 minute, at 90 minute, at 105 minute, at 120 minute and all over the post-operative period

In group $\mathrm{PD}$, the mean of arterial oxygen saturation was $97.2 \pm 0.48 \%$ on admission to operating room, later on there was no significant change in arterial oxygen saturation intraoperatively at 15 minute, at 30 minute, at 45 minute, at 60 minute, at 75 minute, at 90 minute, at 105 minute, at 120 minute and all over the post-operative period

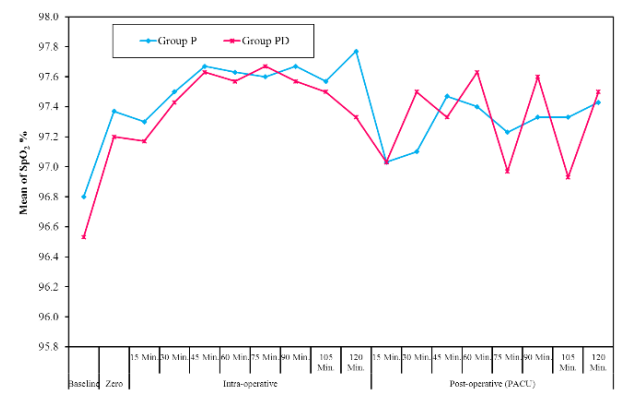

Figure (4): Comparison between the two studied groups according to $\mathrm{SpO} 2 \%$

\section{Changes in the End Tidal Carbon Dioxide ETCO2 (mmHg).}

Regards to ETCO2 group P had a higher average at baseline $(38.13 \mathrm{mmHg}$ vs $36.93 \mathrm{mmHg}$, $\mathrm{p}=0.004)$. Intraoperative the difference between the two groups didn't show statistical difference except at $15,90,105 \mathrm{~min}$, where the average ETCO2 was higher at group $\mathrm{P}$ in compared to group PD

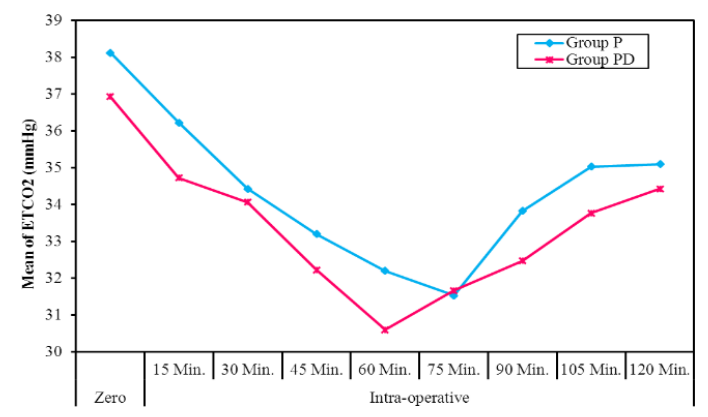

Figure (5): Comparison between the two studied groups according to ETCO2 $(\mathrm{mmHg})$.

Assessment of postoperative pain at rest according to visual analogue scale (VAS).

There was a significant difference as regards to the VAS scores after 1st dose ( $p$ value $<0.001)$, 2nd dose ( $\mathrm{P}$ value $<0.001)$, 3rd dose ( $\mathrm{p}$ value $<0.001)$ and 4 th dose $(\mathrm{p}$ value $<0.001)$.

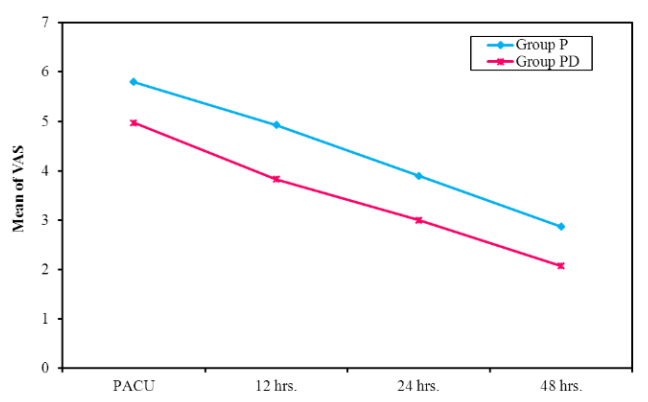

Figure (6): Comparison between the two studied groups according to VAS. 
Comparison between the two studied groups according to analgesic consumption:

Group PD had a significant lower consumption of pethidine in comparison with group $\mathrm{P}$ ( $\mathrm{P}$ value $<0.001)$. However, there was no significant difference observed in the consumption of ketorolac between the two groups $(\mathrm{P}$ value $=0.096)$.

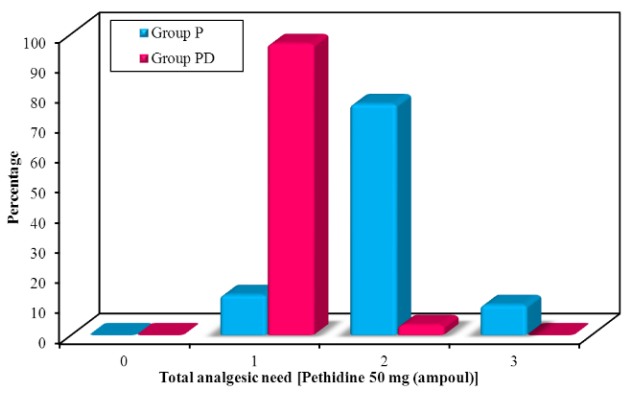

Figure (7): Comparison between the two studied groups according to Total analgesic need [Pethidine $50 \mathrm{mg}$ (ampoule)].

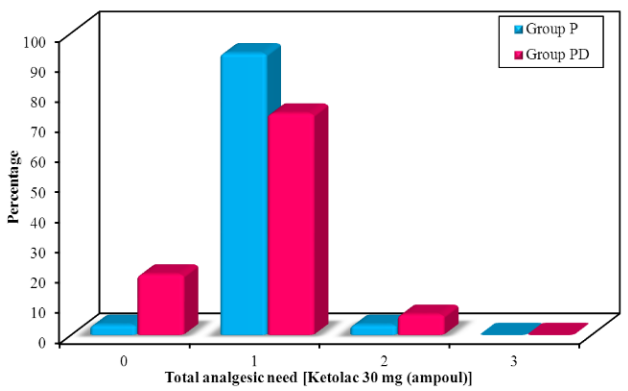

Figure (8): Comparison between the two studied groups according to Total analgesic need [Ketorolac $30 \mathrm{mg}$ (ampoule)].

\section{Postoperative side effects}

There was no significant difference as regards presence of sedation ( $\mathrm{p}$ value $=1$ ), headache $(\mathrm{p} \quad$ value $=0.071)$, visual disturbance $(\mathrm{p}$ value $=0.781)$, nausea $\&$ vomiting $(p$ value $=0.781)$ and the presence of dizziness ( $\mathrm{p}$ value $=0.292$ ).

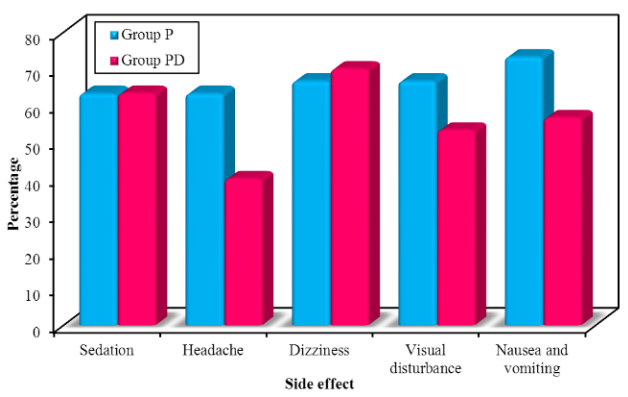

Figure (9): Comparison between the two studied groups according to side effect.
Assessment of patient satisfaction with pregabalin administration using a satisfaction with pain medication (SPM) survey tool:

Total satisfaction was found to be higher in group PD compared to group $\mathrm{P}(88.33 \%$ vs $73.33 \%, \mathrm{P}<0.001)$.

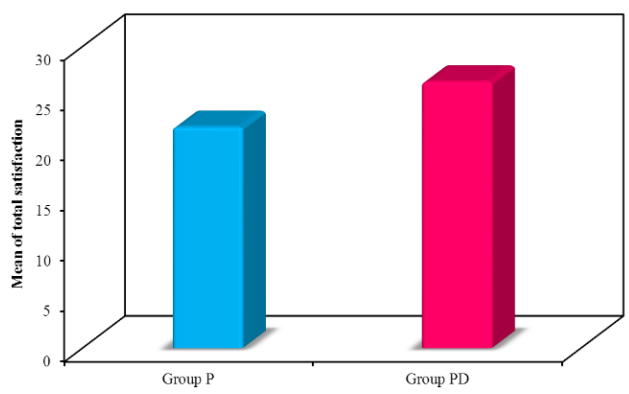

Figure (10): Comparison between the twostudied groups according to Total satisfaction.

Comparison between 2 studied groups according to mobilization time

Regarding mobilization time, group PD exhibited faster mobilization time evident by higher percentage of patients achieving earlier mobilization at $12 \mathrm{hrs}(30 \%$ vs $0 \%)$ and $24 \mathrm{hrs}$ $(43.3 \%$ vs $13.3 \%)$. Furthermore, group $\mathrm{P}$ had higher percentage of patients achieving late mobilization compared to group PD (43.3\% vs $20 \%)$ at $36 \mathrm{hrs}$ and $(43.3 \%$ vs $6.7 \%)$ at $48 \mathrm{hrs}$

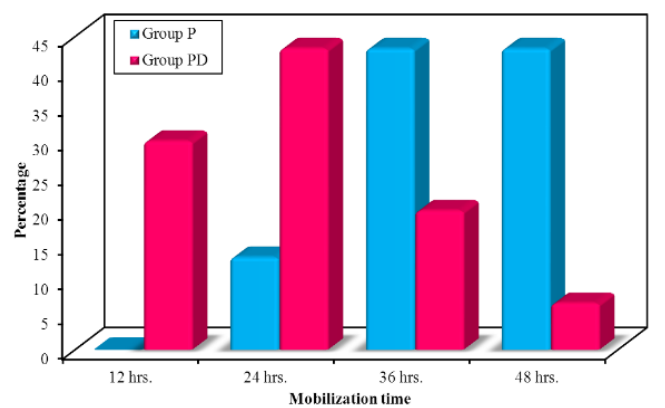

Figure (11): Comparison between the two studied groups according to mobilization time.

\section{DISCUSSION}

Postoperative pain management and treatment is a major challenge in postoperative care and has a critical role in the early mobilization and well-being of the surgical patient.

Results obtained from the present study revealed that there was no significant difference between both groups as regard age, sex and body weight. 
In the present study both groups, as compared with the preoperative base line value, appeared to have no significant change in the HR and MAP. Intra-operatively group PD had values statistically lower than group $\mathrm{P}$ at $15 \mathrm{~min}$ and until the end of surgery; this result suggests that Pregabalin and dexamethasone are effective in preventing hyperactivity of the sympathetic nervous and endocrine systems (neuro-endocrinal reflexes) due to the surgical intervention.

In agreement with our study, Bhawna et al. ${ }^{(5)}$ reviewed the efficacy of oral pregabalin in haemodynamic pressor response of airway instrumentation. A total of 90 normotensive adult consented patients aged 24-56 years, ASA grade I and II, of both gender was randomized into three groups of 30 patients each. The first group received oral placebo, the second group oral pregabalin $75 \mathrm{mg}$ and the third group oral pregabalin $150 \mathrm{mg} 1$ hour prior to induction. The significant increase of haemodynamic pressor response was observed by oral pregabalin premedication in a dose-related manner, with minimum effect on heart rate. The observed increase in haemodynamic values in the control group may be due to inadequate sedation and analgesia ${ }^{(5)}$.

This conclusion came in agreement with that of Bharti et al. ${ }^{(6)}$, where 40 adult females undergoing total mastectomy for breast cancer were included. Patients were randomly allocated to receive pregabalin $(300 \mathrm{mg}$ ) or placebo 2 hours before undergoing the surgery. Patients included in the pregabalin group had lower HR and MAP at pre-induction time in comparison with the control group. This was explained by the attenuated preoperative anxiety effect exerted by pregabalin.

However, in disagreement with the present results, Gupta et al. ${ }^{(7)}$ reviewed the efficacy of oral premedication with pregabalin or clonidine for hemodynamic stability during laryngoscopy and laparoscopic cholecystectomy. A total of 180 patients aged between 35 and 52 years with ASA physical status I and II of both gender, were randomly allocated into 3 groups. The first group received placebo while the second received pregabalin $(150 \mathrm{mg})$ in the second group, and the third group received clonidine $(200 \mu \mathrm{g})$, given 75 to 90 minutes before surgery as oral premedication. Clonidine was superior to pregabalin in attenuation of the hemodynamic responses to laryngoscopy and laparoscopy, but it increased the incidence of intra-and postoperative bradycardia ${ }^{(7)}$.
The present study revealed that the VAS was significantly lower in group PD than in group $\mathrm{P}$ in the post-operative period, in addition the analgesic requirements of pethidine in both groups showed that there was a significant lower consumption of pethidine in group $\mathrm{P}$ than group PD. These results suggest that the peri-operative use of pregabalin and dexamethasone appears to be effective in providing adequate analgesia post-operatively.

This study results showed that single dose of oral pregabalin $(150 \mathrm{mg})$ and intravenous dexamethasone $(16 \mathrm{mg}$ ) was effective in reducing the postoperative pain.

These results are in agreement with Peng et al. ${ }^{(8)}$ testing low dose pregabalin $(75,150 \mathrm{mg})$. Their study showed a significant reduction in postoperative pain intensity assessed by verbal rating score (VRS), which stated that pregabalin is one of the best drugs used for pain control during the immediate postoperative period.

Roth et al. ${ }^{(9)}$ conducted a reviewed a double-blinded, placebo-controlled randomized control trials (RCT) of pregabalin in painful diabetic neuropathy and post-herpetic neuralgia. A total of nine RCTs reported pain as well as sleep measures as end points were included in the review. The review provided data about 2399 patients who were treated two or three times per day with pregabalin (dose 75-600 $\mathrm{mg} /$ day) or placebo on a fixed or flexible schedule. Similar to the current study, Pregabalin at doses $150 \mathrm{mg} /$ day provided a significant analgesic effect as well as better pain-related sleep disturbance ${ }^{(9)}$.

In agreement with the present results, Reuben et al. ${ }^{(10)}$ in a RCT, 80 patients who were undergoing elective spinal fusion surgery received placebo, celecoxib at a dose of $400 \mathrm{mg}$, pregabalin at a dose of $150 \mathrm{mg}$, or a combination of celecoxib at a dose of $400 \mathrm{mg}$ and pregabalin at a dose of $150 \mathrm{mg}$ orally 1 hour before the induction of general anaesthesia. Postoperatively, patients received patient-controlled analgesia (PCA) using morphine. Twelve hours after initial drug administration, patients received a placebo drug, celecoxib at a dose of $200 \mathrm{mg}$, pregabalin at a dose of $150 \mathrm{mg}$, or a combination of celecoxib at a dose of $200 \mathrm{mg}$ and pregabalin at a dose of $150 \mathrm{mg}$ orally. The combination of pregabalin and celecoxib significantly reduced the pain and opioid usage in comparison to the use of either analgesic by itself ${ }^{(10)}$. 
Moreover, the pain reduction was equivocal or sometimes even reduced using a higher pregabalin dose of $300 \mathrm{mg}$, as in those patients undergoing elective abdominal hysterectomy by Ittichaikulthol et al. ${ }^{(11)}$ but on the expense of developing more adverse effects like dizziness and somnolence.

The optimal dosage of pregabalin for attenuating the early post-operative pain management is still debatable in most literature, bouncing in ranges from 50 to $300 \mathrm{mg}$, if safety is also considered. Such variation in dosage was partially attributed to the type of surgery and the nature of pain to be controlled.

Siddiqui et al. ${ }^{(12)}$ studied the effect of a single preoperative oral dose of pregabalin (150 $\mathrm{mg}$ ) in patients with irritable bowel disease who were scheduled for major bowel surgery, and the results suggested that there was no significant reduction in the postoperative pain scores, opioid consumption, or opioid-related side effects.

As regarding the analgesic consumption of pethidine and ketorolac, the current study showed that group $\mathrm{P}$ required higher doses more than group PD significantly.

Zhang et al. ${ }^{(13)}$ reported a simultaneous significant reduction in opioid consumption in the first 24 hours postoperatively when pregabalin was used. This highlights the role of calculating the amount of analgesic consumption, when monitoring the changes in pain intensity.

Similarly, Dalim et al. ${ }^{(13)}$ in another doubleblind RCT compared the effect of the pregabalin 300 $\mathrm{mg}$ or lorazepam $0.5 \mathrm{mg}$ as a pre-medication on the postoperative morphine consumption in 80 women undergoing elective abdominal hysterectomy with/ without salphingo-oophorectomy. Intraoperatively, patients included in the study received intravenous (IV) morphine in a dose of $100-200 \mathrm{~g} / \mathrm{kg}$ and postoperative analgesia was provided with an IV morphine-based PCA regimen: bolus $1 \mathrm{mg}$, lockout interval $5 \mathrm{~min}$, 4-h limit $40 \mathrm{mg}$. Compared to the lorazepam group, patients in the pregabalin group had lower pain scores on the VRS and lower morphine consumption in the first $24 \mathrm{~h}^{(13)}$.

The present study, indicated that there was no detected statistical significant difference between the two studied groups regarding incidence of sedation.

In agreement with the current conclusions Pande et al. (15) found that Pregabalin has an anxiolytic effect. A dose ranging study was conducted to find out the effect of pregabalin as premedication on acute state anxiety and sedation on 108 patients undergoing elective surgeries. The patients were randomly divided to receive any of the following four drugs: placebo, pregabalin in a dose of $75 \mathrm{mg}$, pregabalin in a dose of $150 \mathrm{mg}$, and pregabalin in a dose of $300 \mathrm{mg}$. The level of anxiety, sedation, and pain were assessed at baseline, $30 \mathrm{~min}$, and $60 \mathrm{~min}$ after drug administration, before the induction of anesthesia and every $30 \mathrm{~min}$ in the postanaesthesia care unit (PACU) on an 11-point scale $(0=$ no effect, $10=$ maximal effect). Postoperative fentanyl requirements, PACU and hospital discharge time, early recovery score, and late recovery outcome were assessed. In the "pregabalin $300 \mathrm{mg}$ group", sedation scores were higher compared to the control group during the pre-induction period and at 90 and $120 \mathrm{~min}$ postoperatively. Postoperative fentanyl requirement, and PACU and hospital discharge time were not significantly different among the groups ${ }^{(15)}$.

As regard patient satisfaction, this study showed significantly higher satisfaction scores in group PD more than group P.

In agreement with this observation Jain et al. ${ }^{(16)}$ found that oral pregabalin $75 \mathrm{mg}$ which was given preoperatively to patients undergoing total knee arthroplasty was a useful adjunct to epidural analgesia. It reduced opioid consumption, improves postoperative analgesia, and results in higher patient satisfaction scores ${ }^{(16)}$.

In addition, our study was in line with that reported by Bindu et al. ${ }^{(17)}$ that a single oral dose of pregabalin was effective in reducing the acute postoperative pain in patients undergoing thyroidectomy. It prolongs the time to the request of rescue analgesia and results in lower postoperative pain and higher patient satisfaction scores in the immediate postoperative period. However a statistically significant low opioid consumption could not be proved ${ }^{(17)}$.

Opioids are the mainstay in post-operative pain control. However, due to various side effects associated with high doses (i.e., nausea, vomiting, itching, gastrointestinal and bladder dysfunction, drowsiness, and respiratory depression), a multimodal analgesia approach using a combination of analgesic agents with different mechanism of actions is thought to reduce post-operative opioid consumption and avoid the mentioned side effects which could improve patient's satisfaction and facilitate mobilization and rehabilitation ${ }^{(18)}$. 
In agreement with our results, MensahNyagan et al. ${ }^{(19)}$ reviewed the biological plausibility behind using steroids for pain control; Glucocorticoids can reduce pain by inducing anti-inflammatory actions on the damaged peripheral or central tissue which activates nociceptive mechanisms and generate pain sensation, they are also lipophilic molecules which can cross the blood brain barrier allowing them to work on both the peripheral and central nervous systems ${ }^{(19)}$.

In addition, Bednar et al. ${ }^{(20)}$ reviewed the use of steroids for palliative pain control of the nonoperative patient as epidural and caudal blocks, facet blocks, nerve root blocks etc. A Cochrane review in 2010 by George et al. ${ }^{(21)}$ showed a role for steroids in reducing spine pain from metastatic spinal cord compression. Leppert and Buss ${ }^{(22)}$ reviewed the use of corticosteroids in cancer patients and found that studies suggest they may be effective in the treatment of bone and neuropathic pain when given with opioids. Consequently, dexamethasone a corticosteroid which is commonly used peri-operatively to reduce nausea and vomiting may have a role in postoperative analgesia ${ }^{(22)}$.

Moreover, Wittayapairoj et al. showed that in spinal surgery which is associated with severe acute post-operative pain, intermediate dose of dexamethasone $(16 \mathrm{mg})$ given before anesthetic induction decreased post-operative opioid consumption with no serious side effects. The study attributed these results to the analgesic action of dexamethasone due to inhibition of inflammatory mediators and prevention of pain threshold reduction; also, it reduces tissue swelling and inflammation which prevent nerve compression. The timing of dexamethasone administration remained under debate due to conflicting results between trials, where Jokela et al. demonstrated that administration just before induction can reduce pain, while other studies like those of Waldron et al. ${ }^{(23)}$ showed that pre-operative rather than intra-operative administration is effective in pain reduction. Physiologically, dexamethasone require diffusion through the cell membrane to change gene transcription and produce its effect, this takes about 1-2 hours to occur ${ }^{(23)}$. In conclusion, a single intravenous intermediate dose dexamethasone before induction provided a modest decrease in postoperative opioid consumption within 48 hours of lumbar laminectomy.

Meng and $\mathbf{L i}{ }^{(24)}$ conducted a meta-analysis of RCTs to evaluate the use of dexamethasone in pain control for total joint arthroplasty (TGA) and concluded that dexamethasone could significantly decrease postoperative pain and opioid consumption with the first 48 hours following TGA. However, their study suffered from several limitations as only four RCTs were included with relatively small sample size, dose of dexamethasone varied between studies and duration of follow up was short which may underestimate complications ${ }^{(24)}$.

In a meta-analysis on approximately 2,500 patient performed by De Oliveria et al. (25), intermediate dose dexamethasone was found to have an opioid sparing effect, while also reducing early and late pain, their review also showed that a single dose of dexamethasone had no dose-limiting complications such as wound infection or delayed wound healing, in conclusion, by giving intermediate dose of dexamethasone, beneficial effects on postoperative pain and opioid sparing can be achieved in addition to reduction of nausea and vomiting. A limitation of dexamethasone usage is the occurrence of severe perineal pain, which can be avoided by giving the dose slowly over 10 minutes ${ }^{(25)}$.

\section{CONCLUSION}

Oral pregabalin and intravenous dexamethasone has been demonstrated to have analgesic efficacy after simple lumbar laminectomy. Oral pregabalin and intravenous dexamethasone prevented the increase in $\mathrm{HR}$ and $\mathrm{ABP}$ associated with surgical stimulation. Oral pregabalin and intravenous dexamethasone reduce the analgesic requirements and post-operative opioid use. The use of combined oral pregabalin and intravenous dexamethasone could have higher patient satisfaction. The use of combined oral pregabalin and intravenous dexamethasone allows early mobilization of the patient after simple laminectomy.

\section{REFERENCES}

1. Practice Guidelines for Acute Pain Management in the Perioperative Setting (1995): ASA Task Force on Pain Management. Anesthesiology, 82: 1071.

2. Michael A, Ramsay E (2000): Acute postoperative pain management. BUMC Proceedings, 13: 244-56.

3. Carr DB, Cousins MJ (1998): Spinal route of analgesia: opioids and further options. In: Cousins MJ, Bridenbaugh PO (eds). Neural blockade in clinical anaesthesia and management of pain. 3rd ed. Philadelphia: Lippincott-Raven Publishers.

4. Krebs E, Carey T, Weinberger M (2007): Accuracy of the Pain Numeric Rating Scale as a screening test in primary care. J Gen Intern Med., 22:1453-58. 
5. Bhawna R, Kumkum G, Prashant K, Salony A (2012): Oral Pregabalin premedication for attenuation of haemodynamicpressor response of airway instrumentation during general anaesthesia. Indian J Anaesth., 56(1):49-54.

6. Bharti N, Bala I, Narayan V, Singh G (2013): Effect of pregabaline pretreatment on propofol consumption, hemodynamic variables, and postoperative pain relief in breast cancer surgery. Acta Anaesthesiol Taiwan, 51(1):10-3.

7. Gupta $K$ and Sharma D (2011): Oral premedication with Pregabalin and Clonidine for heamodynamic stability during laryngoscopy and laparoscopic cholecystectomy. Saudi J Anaesth., 5(2)179-84.

8. Peng PWH, Li C, Farcas E, Haley A, Wong $\mathbf{W}$, Bender J et al. (2010): Use of low-dose pregabalin in patients undergoing laparoscopic cholecystectomy. Br J Anaesth., 105(2):155-61.

9. Roth T, van Seventer R, Murphy TK (2010): The effect of pregabalin on pain-related sleep interference in diabetic peripheral neuropathy or postherpetic neuralgia: A review of nine clinical trials. Curr Med Res Opin., 26:2411-9.

10. Reuben SS, Buvanendran A, Kroin JS, Raghunathan K (2006): The analgesic efficacy of celecoxib, pregabalin, and their combination for spinal fusion surgery. Anesth Analg., 103:1271-7

11. Ittichaikulthol W, Virankabutra T, Kunopart M, Khamhom W, Putarawuthichai $\mathbf{P}$, Rungphet S (2009): Effects of pregabalin on post-operative morphine consumption and pain after abdominal hysterectomy with/without salphingo-oophorectomy: a randomized, doubleblind trial. J Med Assoc Thai., 92:1318-23

12. Siddiqui NT, Fischer H, Guerina L, Friedman Z (2014): Effect of a Preoperative pregabalin on Postoperative Analgesia in Patients with Inflammatory Bowel Disease Following Major Bowel Surgery: A Randomized, PlaceboControlled Trial. Pain Practice, 14(2):132-9.

13. Zhang J, Ho KY, Wang Y (2011): Efficacy of pregabalin in acute postoperative pain: a metaanalysis. Br J Anaesth., 106(4):454-62.

14. Dalim KB, Ittichaikulthol W, Virankabutra T, Kunopart M, Khamhom W et al. (2009): Effects of Pregabalin on Post operative Morphine Consumption and Pain after Abdominal Hysterectomy with/without Salphingo-oophorectomy: A Randomized, DoubleBlind Trial. J Med Assoc Thai., 92:1318-23.
15. Pande AC, Feltner DE, Jefferson JW, Davidson JR, Pollack M, Stein MB et al. (2004): Efficacy of the novel anxiolytic pregabalin in social anxiety disorder: A placebocontrolled, multicentre study. J Clin Psychopharmacol., 24:141-9.

16. Jain P, Jolly A, Bholla V, Adatia S, Sood J (2012): Evaluation of efficacy of oral pregabalin in reducing postoperative pain in patientsundergoing total knee arthroplasty. Indian J Orthop., 46(6):646-52.

17. Bindu M, Kumar AA, Kesavan M, Suresh V (2015): Effect of preoperative pregabalin on postoperative pain relief in thyroidectomy patients: A prospective observational study. Anesth Essays Res., 9(2): 161-6.

18. Meng J and Li L (2017): The efficiency and safety of dexamethasone for pain control in total joint arthroplasty. Medicine (Baltimore), 96(24):e7126.

19. Mensah-Nyagan AG, Meyer L, Schaeffer V, Kibaly C, Patte-Mensah C (2009): Evidence for a key role of steroids in the modulation of pain. Psychoneuroendocrinology, 34(1).

20. Bednar DA, Wong A, Farrokhyar F, Paul J (2015): Dexamethasone Perioperative Coanalgesia in Lumbar Spine Fusion: A Controlled Cohort Study of Efficacy and Safety. J Spinal Disord Tech., 28(7):E422-6.

21. George R, Jeba J, Ramkumar G, Chacko Ari G, Tharyan P (2015): Interventions for the treatment of metastatic extradural spinal cord compression in adults. Cochrane Database Syst Rev.,(9).

22. Leppert $W$ and Buss $T$ (2012): The role of corticosteroids in the treatment of pain in cancer patients. Curr Pain Headache Rep., 16(4):307-13.

23. Waldron NH, Jones CA, Gan TJ, Allen TK, Habib AS (2013): Impact of perioperative dexamethasone on postoperative analgesia and side-effects: Systematic review and metaanalysis. Br J Anaesth., 110(2):191-200.

24. Meng J and Li L (2017): The efficiency and safety of dexamethasone for pain control in total joint arthroplasty. Medicine (Baltimore), 96(24):e7126.

25. De Oliveira GS, Almeida MD, Benzon HT, McCarthy RJ (2011): Perioperative Single Dose Systemic Dexamethasone for. Anesthesiology, 115(3):575-88. 